\title{
Hot Topics
}

Z Rheumatol 2017 · 76:195-207

DOI 10.1007/s00393-017-0297-1

(c) Springer-Verlag Berlin Heidelberg 2017

\section{Redaktion}

U. Müller-Ladner, Bad Nauheim

U. Lange, Bad Nauheim

\section{CrossMark}

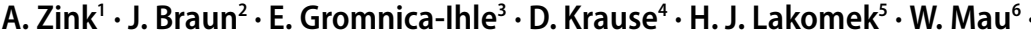 \\ U. Müller-Ladner ${ }^{7,8} \cdot$ J. Rautenstrauch ${ }^{9} \cdot$ C. Specker ${ }^{10} \cdot$ M. Schneider ${ }^{11}$ \\ ' Deutsches Rheuma-Forschungszentrum Berlin, Programmbereich Epidemiologie und Klinik mit \\ Schwerpunkt Rheumatologie und Klinische Immunologie, Charité Universitätsmedizin Berlin, Berlin, \\ Deutschland \\ ${ }^{2}$ Rheumazentrum Ruhrgebiet, Herne, Deutschland \\ ${ }^{3}$ Deutsche Rheuma-Liga Bundesverband, Bonn, Deutschland \\ ${ }^{4}$ Internistische und rheumatologische Gemeinschaftspraxis, Gladbeck, Deutschland \\ ${ }^{5}$ Klinik für Rheumatologie und Interdisziplinäre Geriatrie, Universitätszentrum Innere Medizin, Johannes \\ Wesling Klinikum Minden, Minden, Deutschland \\ ${ }^{6}$ Institut für Rehabilitationsmedizin, Medizinische Fakultät, Martin-Luther-Universität Halle-Wittenberg, \\ Halle, Deutschland \\ ${ }^{7}$ Lehrstuhl für Innere Medizin mit Schwerpunkt Rheumatologie, Justus-Liebig-Universität Gießen, Gießen, \\ Deutschland \\ ${ }^{8}$ Abteilung Rheumatologie und klinische Immunologie, Kerckhoff-Klinik GmbH, Bad Nauheim, \\ Deutschland \\ ${ }^{9}$ Deutsche Gesellschaft für Rheumatologie, Berlin, Deutschland \\ ${ }^{10} \mathrm{Klinik}$ für Rheumatologie \& Klinische Immunologie, Rheumazentrum Rhein-Ruhr e.V., St. Josef \\ Krankenhaus (Universitätsklinikum Essen), Essen, Deutschland \\ "Poliklinik und Funktionsbereich für Rheumatologie, Hiller Forschungszentrum Rheumatologie, \\ Universitätsklinikum Düsseldorf, Düsseldorf, Deutschland
}

\section{Memorandum der Deutschen Gesellschaft für Rheumatologie zur Versorgungsqualität in der Rheumatologie - Update 2016}

\section{Präambel}

Menschen mit einer entzündlich rheumatischen Erkrankung können heute in Deutschland bei rechtzeitiger Diagnosestellung, konsequenter Krankheitskontrolle und angemessener Rehabilitation zumeist ein Leben ohne wesentliche Einschränkungen der Lebensqualität und der sozialen Teilhabe erwarten. Um dies zu erreichen, benötigt das Gesundheitssystem einen optimal abgestimmten Einsatz der verfügbaren diagnostischen und therapeutischen Möglichkeiten in der fachlich gebotenen Qualität entsprechend dem allgemein anerkannten Stand der medizinischen Erkenntnisse ( $\$ 135 \mathrm{a}$ SGB V). Dies soll in ausreichendem, zweckmäßigem und wirtschaftlichem $\mathrm{Maß}$ geschehen ( $\$ 12$ SGB V).

$\mathrm{Zu}$ einer Versorgung in der fachlich gebotenen Qualität gehören das frühzei- tige Erkennen von Verdachtsfällen, die fachärztliche Untersuchung und Versorgung von Neuerkrankten innerhalb von 3 Monaten nach Symptombeginn sowie die kontinuierliche und konsequent zielorientierte Behandlung von Menschen mit entzündlich rheumatischen Erkrankungen in einem Versorgungsnetzwerk unter der Leitung eines Facharztes für innere Medizin und Rheumatologie. Weitere Elemente einer qualitativ angemessenen Versorgung sind die Patientenschulung als Basis einer partizipativen Entscheidungsfindung und die fachspezifische stationäre Versorgung im Bedarfsfall. Diese Versorgung muss in Deutschland jedem Betroffenen zur Verfügung stehen, unabhängig von Alter, Geschlecht, Wohnregion, Sozialstatus und ethnischer Zugehörigkeit.

Mit der hier vorgelegten Aktualisierung des Memorandums der Deutschen
Gesellschaft für Rheumatologie (DGRh) „Rheumatologische Versorgung von akut und chronisch Rheumakranken in Deutschland" [5] aus dem Jahr 2008 werden die derzeit in Deutschland bestehenden Voraussetzungen für eine den wissenschaftlichen Erkenntnissen entsprechende Versorgung von Menschen mit entzündlich rheumatischen Erkrankungen untersucht und bewertet. In dem Memorandum von 2008 wurde ausführlich der Bedarf an interdisziplinärer rheumatologischer Versorgung von Erwachsenen in Deutschland ermittelt, dargestellt und diskutiert. Im Jahr 2016 besteht dieser Versorgungsbedarf im Grundsatz unverändert weiter. Deutlich gewandelt haben sich jedoch die Therapieoptionen, die Therapiestrategien („treat-to-target“) und die erreichbaren Therapieziele (Remission). Dies hat Auswirkungen auf den rheumatologi- 
schen Versorgungsbedarf. Der Vorstand der Deutschen Gesellschaft für Rheumatologie hat daher Anfang 2016 die Kommission Versorgungsqualität damit beauftragt, eine Aktualisierung des Memorandums vorzunehmen. Aufgabe war es, den Versorgungsbedarf auf dem Gebiet der internistisch rheumatologischen Versorgung $\mathrm{zu}$ definieren. Es bestand nicht der Anspruch, den Versorgungsbedarf auf dem Gebiet der Kinder- und Jugendrheumatologie, der nichtärztlichen Heilberufe oder der operativen Fächer zu ermitteln.

Die Kommission bestand aus einer multidisziplinär zusammengesetzten Gruppe von Rheumatologen in der ambulanten vertragsärztlichen, universitären, akutstationären und rehabilitativen Versorgung, Epidemiologen sowie Vertretern der Deutschen Rheuma-Liga und der Deutschen Gesellschaft für Rheumatologie.

Dieses Memorandum legt Anhaltszahlen zu einer bedarfsgerechten Versorgung sowie Empfehlungen für notwendige Verbesserungen vor. Der Schwerpunkt liegt auf der Berechnung der erforderlichen personellen Ressourcen, insbesondere der Zahl der in der Versorgung tätigen internistischen Rheumatologen und der optimalen Nutzung der vorhandenen Kapazitäten. Im Einklang mit den bestehenden Strukturen unserer Gesundheitsversorgung wird eine Bedarfsermittlung vorgenommen, die auf den Zahlen der betroffenen Menschen, dem in der Versorgung erforderlichen Zeitaufwand und den vorhandenen Angebotsstrukturen basiert. Dabei wird den gesetzlichen Vorgaben nicht nur hinsichtlich der Qualität, sondern auch hinsichtlich der Wirtschaftlichkeit Rechnung getragen.

Der internistische Rheumatologe hat die spezifische Aufgabe, die Versorgung von Personen mit rheumatischen und muskuloskeletalen Erkrankungen, insbesondere mit entzündlich rheumatischen Gelenkerkrankungen, inflammatorischen/immunologischen Systemerkrankungen, endokrinen und metabolischen Erkrankungen mit rheumatischer Symptomatologie sowie schweren Verlaufsformen anderer muskuloskeletaler Erkrankungen verantwortlich zu leiten, zu steuern und zu begleiten. Seine
Qualifikation erlaubt es ihm, komplexe Krankheitsbilder mit vielfältigen Organbeteiligungen, einem erhöhten Risiko lebensbedrohlicher Komorbiditäten und den möglichen Komplikationen etablierter und innovativer immunsuppressiver Therapien optimal zu versorgen. Der internistische Rheumatologe handelt in Abstimmung mit dem Hausarzt und mit fallspezifisch hinzuzuziehenden Vertretern weiterer ärztlicher Fachrichtungen, nichtärztlichen Heilberufen (v. a. Physiotherapeuten, Ergotherapeuten, Orthopädietechnikern und Psychologen bzw. Psychotherapeuten) sowie Patientenorganisationen.

Im Folgenden wird der Einfachheit halber die männliche Form bei Betroffenen und Ärzten verwendet. Gemeint sind damit immer beide Geschlechter.

\section{Aufgaben und Leistungen des internistischen Rheumatologen}

Internistische Rheumatologen sind Fachärzte für innere Medizin und Rheumatologie. Im Rahmen ihrer mindestens 6 Jahre dauernden Facharztweiterbildung haben sie sich Kenntnisse, Erfahrungen und Fertigkeiten in der Erkennung und Behandlung rheumatischer und muskuloskeletaler Erkrankungen erworben. Hierzu gehören Kenntnisse und Fertigkeiten hinsichtlich der speziellen Anamnese bei internistischen und immunologischen Erkrankungen, der klinischen internistischen und rheumatologischen Untersuchungstechniken, der intraartikulären Punktionen, der rheumatologisch immunologischen Labordiagnostik, der radiologischen Diagnostik einschließlich der Magnetresonanztomographie (MRT) und der Sonographie von Bewegungsorganen und Blutgefäßen.

\section{Ambulante rheumatologische Versorgung}

Die Bedarfsplanungsrichtlinie des Gemeinsamen Bundesausschusses definierte 2015 als Grundstruktur der Bedarfsplanung 4 Versorgungsebenen: die hausärztliche Versorgung, die allgemeine fachärztliche Versorgung, die spezialisierte fachärztliche Versorgung sowie die gesonderte fachärztliche Versorgung [9]. Auf der Ebene der spezialisierten fachärztlichen Versorgung sind fachärztlich tätige internistische Rheumatologen für die ambulante Versorgung von Patienten mit den in der Präambel beschriebenen rheumatischen und muskuloskeletalen Erkrankungen zuständig. Diese können in rheumatologischen Schwerpunktpraxen, medizinischen Versorgungszentren, ermächtigten Rheumaambulanzen, Universitätsambulanzen, Rheumaambulanzen nach $₫ 116 \mathrm{~b}$ (alt) oder zukünftig auch in der ambulanten spezialfachärztlichen Versorgung (ASV) arbeiten.

Die Zuweisung eines Patienten zum internistischen Rheumatologen durch Hausärzte oder andere Fachärzte erfolgt zur Erstuntersuchung und Diagnosestellung bei Verdacht auf eine rheumatische Erkrankung oder zur Behandlung bzw. Mitbehandlung von Patienten mit bereits festgestellter Erkrankung sowie von jungen Erwachsenen mit einer im Kindesalter aufgetretenen Rheumaerkrankung (Transition).

Bei Patienten, die sich zur Erstuntersuchung vorstellen, besteht die Aufgabe des internistischen Rheumatologen in einer möglichst zeitnah durchgeführten, umfassenden Differenzialdiagnostik, bei der, ausgehend von der Krankheitsvorgeschichte und dem klinischen Befund, u. a. radiologische, sonographische und labormedizinische Methoden eingesetzt werden.

In aller Regel verlaufen entzündlich rheumatische Erkrankungen chronisch und bedürfen der langfristigen Betreuung durch den internistischen Rheumatologen. Dieser übernimmt die Koordination bei komplexen Therapien oder komplizierten Verläufen in $\mathrm{Zu}$ sammenarbeit mit dem Hausarzt und den anderen in die Behandlung eingebundenen Ärzten sowie Angehörigen anderer Gesundheitsfachberufe. Der Patient wird mit der Zielsetzung einer partizipativen Entscheidungsfindung als gleichberechtigter Partner in den Entwurfdes Behandlungsplanes einbezogen, seine Motivation und Eigenverantwortung werden gezielt angesprochen. Der Behandlungsplan ist individuell auf den Patienten zugeschnitten und wird mit diesem hinsichtlich der Art der Erkran- 
Z Rheumatol 2017· 76:195-207 DOI 10.1007/s00393-017-0297-1

c) Springer-Verlag Berlin Heidelberg 2017

A. Zink · J. Braun · E. Gromnica-Ihle · D. Krause · H. J. Lakomek · W. Mau · U. Müller-Ladner · J. Rautenstrauch · C. Specker · M. Schneider

\section{Memorandum der Deutschen Gesellschaft für Rheumatologie zur Versorgungsqualität in der Rheumatologie - Update 2016}

\section{Zusammenfassung}

Im Auftrag des Vorstands der Deutschen

Gesellschaft für Rheumatologie (DGRh)

hat die interdisziplinär zusammengesetzte

Kommission Versorgungsqualität 2016

eine Aktualisierung des Memorandums zur

rheumatologischen Versorgung von 2008 vor-

genommen. Die Aktualisierung berücksichtigt

die veränderten Therapiestrategien und

-ziele sowie aktuelle Versorgungsstrukturen

und politische Rahmenbedingungen.

Schwerpunkt ist die Ermittlung des Bedarfs

an internistischen Rheumatologen in

der ambulanten, akutstationären und

rehabilitativen Versorgung. Der internistische

Rheumatologe ist primär zuständig für die

Versorgung von Personen mit entzündlich

rheumatischen Systemerkrankungen. Je

100.000 erwachsene Einwohner werden

mindestens 2 internistische Rheumatologen

in der ambulanten Versorgung gebraucht, dies

sind 1350 Rheumatologen. Mit derzeit 776 internistischen Rheumatologen fehlt fast die Hälfte des Mindestbedarfs. Die DGRh fordert eine eigene ambulante Bedarfsplanung für internistische Rheumatologen, um das Versorgungsdefizit abzubauen. Die akutstationäre Versorgung sollte in spezialisierten Akutkliniken und -abteilungen erfolgen, die eine hohe Behandlungsqualität der komplexen Krankheitsbilder sicherstellen. Es sollten bis zu 50 Betten je 1 Mio. Einwohner vorgehalten werden. Je 30 Betten werden mindestens 2 hauptamtlich stationär tätige internistische Rheumatologen und 3 weitere ärztliche Mitarbeiter benötigt. Im Bereich der Rehabilitation der Kranken- und Rentenversicherung sollten je 1 Mio. Einwohner 40 Betten oder ambulante Plätze mit jeweils 1 hauptamtlich tätigen Rheumatologen und 1 weiteren ärztlichen Mitarbeiter zur Verfügung stehen. Um die bestehenden Defizite abzubauen und den absehbar wachsenden
Bedarf an internistischen Rheumatologen zu decken, sind verstärkte Anstrengungen im Bereich der ärztlichen Ausbildung durch Schaffung rheumatologischer Lehrstühle an jeder medizinischen Fakultät sowie im Bereich der Weiterbildung erforderlich. In allen Versorgungssegmenten sind die Behandlungsziele gemeinsam mit den Betroffenen im Sinne partizipativer Entscheidungsfindung festzulegen. Anzustreben ist die Versorgung in einem interdisziplinären Netzwerk unter Einbindung anderer ärztlicher Fachrichtungen, nichtärztlicher Heilberufe sowie Patientenorganisationen.

\section{Schlüsselwörter}

Muskuloskeletale Erkrankungen · Ausbildung $\cdot$ Ambulante Versorgung $\cdot$ Stationäre Versorgung $\cdot$ Rehabilitation

\section{Memorandum of the German Society for Rheumatology on the quality of treatment in rheumatology - Update 2016}

\section{Abstract}

On behalf of the Steering Committee of the German Society for Rheumatology, in 2016 the Interdisciplinary Commission on Healthcare Quality updated the 2008 memorandum on rheumatological healthcare in Germany. The update considers changes in therapeutic strategies, treatment targets as well as current structures in healthcare and the political framework. It concentrates on examination of the need for rheumatologists with a background in internal medicine and determines the gap between needs and supply. The internist rheumatologist is responsible for the care of patients with inflammatory rheumatic diseases and contributes to the care of patients with severe forms of other musculoskeletal diseases. At least 2 internist rheumatologists are needed for the outpatient care of 100,000 adult inhabitants, equivalent to 1350 rheumatologists in Germany. With currently 776 rheumatologists, we have little more than half of what we need. The German Society for Rheumatology calls for specific requirements planning for rheumatologists in outpatient care in order to decrease the deficit. In acute inpatient care we need specialized hospitals and wards that ensure a high quality of treatment for patients with complex diseases. We need up to 50 beds per 1 million inhabitants. At least 2 full-time internist rheumatologists and 3 further physicians are needed per 30 beds. In inpatient and outpatient rehabilitation we need 40 beds or outpatient places per 1 million inhabitants with at least 1 full-time rheumatologist and 1 further physician. In order to reduce the existing deficits and to cover the increasing future need for rheumatologists, more emphasis has to be laid on primary and secondary education. Chairs for internal rheumatology are needed at each medical university and more positions for postgraduate training in rheumatology should be provided. In all segments of healthcare the treatment aims should be jointly defined between patients and physicians. The patients should be treated in an interdisciplinary network, comprising other medical specialties, health professionals as well as patient organizations.

\section{Keywords}

Musculoskeletal diseases - Education .

Outpatient care $\cdot$ Inpatients · Rehabilitation kung, der eingeleiteten Therapien, der Kontrolluntersuchungen und der möglichen Auswirkungen auf die weitere Lebensgestaltung erörtert. Der Behandlungsplan enthält neben medikamentösen Therapien auch Empfehlungen zur Lebensführung, ggf. die Verordnung von Heil- und Hilfsmitteln bzw. die Einbeziehung einer orthopädischen, neurologischen, schmerztherapeutischen oder einer anderen fachärztlichen Mitbehandlung. Gespräche hierüber und über Selbsthilfe (ggf. mit Anschluss an entsprechende Organisationen) stellen ebenso wie Stellungnahmen zu sozialmedizinischen Fragen gewichtige und zeitaufwendige Säulen in der ambulanten Versorgung Rheumakranker dar.

In Abhängigkeit vom Krankheitsverlauf und der Erreichung der festgelegten Therapieziele werden die Kontrollin- 
tervalle modifiziert. Diese können wenige Wochen, aber auch mehrere Monate bis hin zu 1 Jahr betragen. Insbesondere werden für akute Zustandsverschlechterungen kurzfristige Termine zur Akutbehandlung und Umstellungen des Behandlungsplanes bereitgestellt.

Eine qualitativ hochwertige ambulante Versorgung hat daher folgende Voraussetzungen:

- Primär versorgende Ärzte (Allgemeinmediziner, Orthopäden etc.) müssen über grundlegendes rheumatologisches Wissen verfügen, um Patienten mit Bedarf an rheumatologischer Mitbetreuung sicher von solchen ohne diesen Bedarf zu unterscheiden.

- Ein schneller Zugang zum internistischen Rheumatologen zur Diagnoseklärung muss für alle Betroffenen gesichert sein (z. B. durch Früherkennungssprechstunden).

- Hat der internistische Rheumatologe eine entzündlich rheumatische Krankheit festgestellt, so wird er den Patienten entsprechend informieren, die Therapie einleiten und im Bedarfsfall auch die Heil- und Hilfsmittelversorgung vornehmen sowie die Indikation zur Rehabilitation stellen.

- Bei den meisten entzündlich rheumatischen Erkrankungen ist eine Überwachung des Verlaufs durch den internistischen Rheumatologen in regelmäßigen Abständen erforderlich. Hierbei wird die Krankheitsaktivität erfasst und die Therapie im Hinblick auf ihre Wirkung und evtl. unerwünschte Wirkungen überwacht und angepasst. Auswirkungen der Krankheit auf das tägliche Leben und die Teilhabe sowie die Diagnose, Prognose und Therapie werden in patientengerechter Form thematisiert und durch eine gezielte Patienteninformation oder -schulung ergänzt. Im Ergebnis werden mehrdimensionale Therapieziele und -pläne zwischen Patienten und Rheumatologen vereinbart.

- Individuelle medizinische Informationen (Therapieplan, Berichte, Bildgebung, Laborwerte etc.) sollten für die an der Versorgung Beteiligten durch eine geeignete informationstechnologische (IT-)Infrastruktur in angemessener Weise zugänglich sein.

Modellhaft wurde dies in den bundesweit verhandelten Verträgen der Versorgungslandschaft Rheuma umgesetzt, einem gemeinsam von Hausärzten und internistischen Rheumatologen entwickelten sektorenübergreifenden Ansatz [1]. Erste Auswertungen der Daten der Versorgungslandschaft Rheuma zeigen, dass etwa $25 \%$ der Patienten im Rahmen von Früherkennungssprechstunden versorgt werden und eine hohe Patientenzufriedenheit erreicht wird. Analoge, meist weniger umfassende Verträge bestehen regional in einzelnen Bundesländern und wurden unter Mitarbeit von internistischen Rheumatologen in der Regel zwischen einzelnen Krankenkassen und Kassenärztlichen Vereinigungen geschlossen.

\section{Stationäre rheumatologische Versorgung}

Die Indikation zur akutstationären Aufnahme besteht zur diagnostischen Abklärung einer komplexen Krankheitssymptomatik oder zur Therapie von schweren Erkrankungen einschließlich ihrer krankheits- oder therapiebedingten Komplikationen und Folgen, falls dies ambulant nicht geleistet werden kann. Mögliche Indikationen können sein:

- hohe Krankheitsaktivität bzw. akuter Schub der Erkrankung,

- akute Therapiekomplikationen [23],

- Organbeteiligungen [18],

- starke akute oder chronische Schmerzen,

- akute Funktionseinschränkungen.

Rheumatologische Akutkliniken halten entsprechende Ressourcen vor, um Rheumapatienten mit komplexen Krankheitsverläufen mit einer hohen Behandlungsqualität zeitnah und adäquat zu versorgen. Wichtige Elemente der Strukturqualität sind die 24-stündige Erreichbarkeit und der Facharztstatus auch bei der Notfallversorgung.

Den besonderen Herausforderungen in der Versorgung chronisch betroffener Patienten mit einer häufig kom- plexen Krankheitssymptomatik wird für die akutstationäre Rheumatologie durch eine sachgerechte Kalkulation im G-DRG-System Rechnung getragen [8, 22]. Durch die Mitgliedskliniken des Verbands rheumatologischer Akutkliniken wurden seit Anfang dieses Jahrhunderts Eckpfeiler zur Qualitätssicherung (Struktur-, Prozess- und Ergebnisqualität) in den klinischen Einrichtungen implementiert, die dazu beitragen, die Patientenversorgung auf hohem Qualitätsniveau umzusetzen. Ein Leitprojekt ist das bereits 2003 begonnene und von der Rheuma-Liga mit entwickelte KOBRA-Projekt (kontinuierliches Outcome-Benchmarking in der rheumatologischen Akutversorgung), an dem derzeit 25 Kliniken teilnehmen [17]. Es hat wichtige Impulse für eine patientenfokussierte akutstationäre Rheumaversorgung gesetzt [3]. Das KOBRA-Projekt ist im internationalen Vergleich das einzige Qualitätsprojekt in der akutstationären rheumatologischen Versorgung, das auch die Ergebnisqualität misst.

Um die erforderliche stationäre Versorgungsqualität sicherstellen zu können, benötigt eine Rheumaklinik/-abteilung mit durchschnittlich 30 Betten

- mindestens 2 hauptamtlich im stationären Bereich tätige internistische Rheumatologen (1 Chefarzt und 1 Oberarzt). Dies gewährleistet den multidisziplinären Behandlungsansatz unter fachärztlicher Leitung, der bereits 2005 mit der multimodalen rheumatologischen Komplexbehandlung (OPS-8-983) Eingang in den Operationen- und Prozedurenschlüssel (OPS) gefunden hat $[20,21]$. Seit 2006 findet sich im DRG-System die Ziffer I97Z, die für die rheumatologische Komplexbehandlung durch ein multiprofessionelles Team bei einer Mindestverweildauer von 14 Tagen steht,

- mindestens 3 weitere stationär tätige ärztliche Mitarbeiter (einschließlich Weiterbildungsassistenten) in Abhängigkeit von der Struktur der Einrichtung. Für zusätzliche Aufgaben wie ambulante Versorgung, Konsile, Lehre, Forschung etc. sind weitere ärztliche Mitarbeiter erforderlich. Patienten, die wegen einer 
Hier steht eine Anzeige.

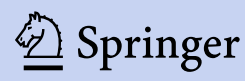


entzündlich rheumatischen Erkrankung stationär behandelt werden, sollten diese Behandlung nur in dafür fachlich qualifizierten Rheumakliniken/-abteilungen erfahren, um die notwendige Behandlungsqualität $\mathrm{zu}$ sichern [16].

Nicht selten wird in den rheumatologischen Akutkliniken auch geriatrische und schmerztherapeutische Expertise vorgehalten, um entsprechende Gesundheitsprobleme adäquat lösen $\mathrm{zu}$ können.

\section{Medizinische rheumatologische Rehabilitation}

Die Kompetenzen und Tätigkeiten internistischer Rheumatologen in Einrichtungen zur medizinischen Rehabilitation zielen auf die Verbesserung und den Erhalt der funktionalen Gesundheit von Menschen mit insbesondere entzündlich rheumatischen Krankheiten [26]. Aufgrund des Systemcharakters rheumatischer Krankheiten sowie der zahlreichen Komorbiditäten ist internistische Fachkompetenz auch für die rheumatologische Rehabilitation unerlässlich. Ausgangsbasis ist zunächst die im Einzelfall aufwendige systematische Erfassung der vielfältigen Einschränkungen und der verbliebenen Ressourcen von Rheumakranken. Hierzu dient in erster Linie die internationale Klassifikation der Funktionsfähigkeit, Behinderung und Gesundheit (ICF) der Weltgesundheitsorganisation.

Gemäß den gesetzlichen Rahmenbedingungen stellen sich dem internistischen Rheumatologen in Zuweisung und Durchführung der Rehabilitation Rheumakranker 2 Aufgaben [5]:

1. die Erwerbsfähigkeit wiederherzustellen oder zu erhalten („Rehabilitation vor Rente “ - Leistungsträger v. a. Gesetzliche Rentenversicherung [GRV] nach Sozialgesetzbuch VI) oder

2. Behinderung und Pflegebedürftigkeit zu vermeiden oder deren Ausmaß zu vermindern („Rehabilitation vor Pflege“ - Leistungsträger v. a. Gesetzliche Krankenversicherung [GKV] nach Sozialgesetzbuch V).
Folgende Patientengruppen mit entzündlich rheumatischen Krankheiten sind für die zum Teil unterschiedlich fokussierten rehabilitativen Interventionen zu unterscheiden [26]:

- „Frühfälle“ mit drohender oder beginnender Funktionseinschränkung (v. a. Krankheitsinformation, Gelenkschutz, Prävention von Schüben und Folgeschäden, Schulung und Training durch ein spezialisiertes Team),

- Patienten mit manifesten körperlichen Funktionseinschränkungen ( $\mathrm{Pa}-$ tientenschulung, Funktionstraining, Verbesserung von Koordination, Kraft und Ausdauerleistung),

- Patienten mit zusätzlichen psychosozialen Belastungen (Entspannungstechniken, Schmerzbewältigungsund Coping-Strategien, psychologische Interventionen, Partnergespräche),

- Patienten mit besonderen beruflichen Problemlagen (akzentuierte medizinisch beruflich orientierte Rehabilitation mit vergleichenden Analysen der arbeitsplatzbezogenen Fähigkeits- und Anforderungsprofile sowie darauf abgestimmten Interventionen).

$\mathrm{Zu}$ den Aufgaben des internistischen Rheumatologen in der medizinischen rheumatologischen Rehabilitation gehören

- unter der Zielstellung ,treat to participation" gemeinsam mit den Betroffenen die Definition von Rehabilitationszielen entsprechend dem individuellen Problem- und Risikoprofil [26],

- sozialmedizinische Begutachtungen mit Erstellung eines qualitativen und quantitativen Leistungsbildes bei allen medizinischen Rehabilitationsleistungen in Trägerschaft der DRV,

- die Einleitung bzw. konkrete Empfehlung einer systematischen Rehabilitationsnachsorge und insbesondere die Weichenstellung zur Durchführung von differenzierten Leistungen zur Teilhabe am Arbeitsleben (früher als berufliche Rehabilitation bezeichnet),
- eine differenzierte Verlaufsprognose unter Berücksichtigung der Komorbiditäten,

- die Koordination eines multimodalen, trans- und interdisziplinären Krankheitsmanagements im Team aus Physio-, Sport-, Ergotherapeuten, Psychologen, Ernährungsberatern, Pflegekräften, Orthopädie(Schuh-)Technikern sowie RehaFachberatern/Sozialarbeitern,

- die Befähigung der betroffenen Patienten zum eigenverantwortlichen Krankheitsmanagement über standardisierte krankheitsspezifische Patientenschulungen der DGRh [30].

\section{Bedarf und bedarfsgerechte Versorgung aus Patientensicht}

Bei allen gesundheitspolitischen Prozessen ist die Patientenperspektive $\mathrm{zu}$ berücksichtigen. Der „Aufbruch in das Jahrhundert des Patienten “ [11] ist notwendige Voraussetzung einer weiteren Professionalisierung der modernen $\mathrm{Me}$ dizin. Die Beteiligung von Betroffenen erstreckt sich nicht nur auf die direkte Arzt-Patienten-Kommunikation, sondern auch grundsätzlich auf gesundheitspolitische Entscheidungsprozesse.

Ein Patient mit Gelenkschmerzen oder anderen Symptomen einer entzündlich rheumatischen Erkrankung benötigt den unmittelbaren Zugang zu seinem Hausarzt. Dieser muss ein rheumatologisches Basiswissen aufweisen, um eine erste Sichtung und ggf. Überweisung vornehmen zu können. Hat der primär versorgende Arzt den begründeten Verdacht auf eine entzündlich rheumatische Erkrankung, sollte der Zugang zum internistischen Rheumatologen innerhalb von 2 Wochen gewährleistet sein.

Aus der Perspektive der Betroffenen sind bei der Einschätzung der Erkrankung nicht nur klinische Parameter wie Gelenkbeteiligung, Entzündungswerte oder eine bildgebend nachgewiesene Destruktion der Gelenke bedeutsam, sondern auch die damit im Zusammenhang stehenden Symptome, die das tägliche Leben und die Teilhabe einschränken. „Patient-Reported Outcomes“ (PROs) wie Schmerz, Funktionsfähigkeit, Fatigue oder Erwerbstätig- 
keit/Arbeitsfähigkeit sind entscheidende Parameter, die den Einfluss der rheumatischen Erkrankung auf das Leben der Betroffenen widerspiegeln und seine gesundheitsbezogene Lebensqualität abbilden. Diese werden mit klinischen und Laborparametern allein nicht erfasst.

Die Behandlungsziele sind gemeinsam mit den Betroffenen im Sinne einer partizipativen Entscheidungsfindung festzulegen, entsprechend deutschen und internationalen Therapieempfehlungen [2]. Der idealerweise gemeinsam zwischen Arzt und Patient erstellte Behandlungsplan sollte, wie auch alle anderen Befunde, allen involvierten Behandlern in einem Netzwerk zur Verfügung stehen. Aus Sicht der Betroffenen sind bei dieser Planung alle Therapieformen wie ausreichende physikalische Therapie einschließlich notwendiger Ergotherapie, evtl. psychologische Betreuung, Schmerztherapie, die Versorgung mit Hilfsmitteln guter Qualität genauso einzubeziehen wie Maßnahmen zur Information und Schulung des Patienten.

\section{Ambulanter, akutstatio- närer und rehabilitativer Versorgungsbedarf}

Schwerpunkte der Versorgungsleistung internistischer Rheumatologen sind die Abklärung und Behandlung entzündlich rheumatischer Systemerkrankungen, also z. B. der rheumatoiden Arthritis, der Spondyloarthritiden, Kristallarthropathien, Kollagenosen und Vaskulitiden sowie autoinflammatorischer Syndrome. Die Prävalenzen dieser Krankheitsbilder addieren sich auf etwa 2,1\% der erwachsenen Bevölkerung oder 1,45 Mio. Betroffene [32]. Darüber hinaus müssen internistische Rheumatologen über profunde differenzialdiagnostische Kenntnisse anderer muskuloskeletaler Krankheitsbilder und Komorbiditäten, wie z. B. Arthrosen, Osteoporose oder Fibromyalgie, verfügen.

\section{Ambulante Versorgung}

Die Kommission geht - wie im Memorandum von 2008 näher ausgeführt - weiterhin davon aus, dass Patienten mit bereits diagnostizierter entzündlich rheumatischer Erkrankung in der ambulanten Versorgung im Mittel 4-mal pro Jahr rheumatologisch gesehen werden müssen und dass jede Konsultation einschließlich Vor- und Nachbereitung durchschnittlich 20 min dauert. Der Bedarf an ambulanter internistisch rheumatologischer Versorgung von 100.000 Erwachsenen lässt sich hieraus wie folgt berechnen: 2100 Fälle $\times 4$ Kontakte $\times$ $20 \mathrm{~min}$ je Fall $=2800 \mathrm{~h}$.

Zusätzlich $\mathrm{zu}$ berücksichtigen sind die inzidenten Fälle der genannten Erkrankungen, deren Abklärung im Mittel 1,5 Kontakte à $40 \mathrm{~min}$ (d. h. $1 \mathrm{~h}$ pro Patient und Jahr) erfordert. Unterstellen wir eine durchschnittliche Krankheitsdauer von 20 Jahren, ergäben sich pro 100.000 Einwohner und Jahr 105 solcher Fälle. Ihre Versorgung würde noch einmal $105 \mathrm{~h}$ erforderlich machen.

Weiter zu berücksichtigen sind Kranke mit schweren, prognostisch ungünstigen Verlaufsformen von Arthrosen, chronischen Schmerzsyndromen der Bewegungsorgane sowie Osteopathien. Hier stehen Differenzialdiagnostik und Behandlungsempfehlungen im Vordergrund. Die Schwerpunktbetreuung dieser Patienten kann in der Regel anderen ärztlichen Disziplinen überantwortet werden. Etwa 18 Mio. Menschen in Deutschland (26.000 je 100.000 Erwachsene) sind von diesen Krankheiten betroffen [32]. Für diese Patienten muss im Bedarfsfall die grundsätzliche Möglichkeit der konsiliarischen Mitbetreuung durch einen internistischen Rheumatologen bestehen. Wenn wir bei $10 \%$ dieser Betroffenen einen rheumatologischen Mitbetreuungsbedarf von lediglich $15 \mathrm{~min}$ pro Patient und Jahr veranschlagen, so ergibt sich je 100.000 Einwohner ein jährlicher Gesamtzeitbedarf von $2600 \times 0,25 \mathrm{~h}=650 \mathrm{~h}$.

Zusammen genommen ergibt sich hieraus pro 100.000 Erwachsene und Jahr ein Zeitbedarf von 3555 Arbeitsstunden internistischer Rheumatologen. Das ZI-Panel von 2014 [31] hat bei Internisten eine mittlere Zahl von 54 Arbeitsstunden pro Woche, davon $38 \mathrm{~h}$ in der direkten Patientenversorgung, ermittelt, an 224 Tagen pro Jahr (1702 h pro Jahr). Unter Zugrundelegung dieser sehr hohen Arbeitsleistung ergibt sich ein Mindestbedarf von 2 internistischen Rheumatologen je 100.000 erwachsene Einwohner oder 1350 Rheumatologen bei 68 Mio. Erwachsenen (• Tab. 1).

Der ermittelte Bedarf wird deutlich höher, wenn eine durchschnittliche Gesamtarbeitszeit von $40 \mathrm{~h}$ pro Woche (davon wie in der ZI-Berechnung $70 \%$ in der direkten Patientenversorgung $=28 \mathrm{~h}$ ) zugrunde gelegt würde (1254 Arbeitsstunden pro Jahr). Zudem haben nicht alle vertragsärztlich tätigen Rheumatologen einen vollen Versorgungsauftrag. Dies trifft auf $70 \%$ der hausärztlich und $67 \%$ der fachärztlich niedergelassenen Rheumatologen $\mathrm{zu}$ [15]. Weitere $11 \%$ der hausärztlich und $10 \%$ der fachärztlich tätigen Rheumatologen haben einen Versorgungsauftrag von mehr als $30 \mathrm{~h}$ pro Woche. Die übrigen $19 \%$ der hausärztlich und $23 \%$ der fachärztlich tätigen Rheumatologen haben Versorgungsaufträge zwischen 10 und $30 \mathrm{~h}$. Man müsste also einen Abschlag von mindestens $10 \%$ der Arbeitszeit vornehmen (entspricht 1130 leistbaren Arbeitsstunden pro Jahr). Unter diesen Prämissen ergibt sich ein Bedarf von 3 internistischen Rheumatologen je 100.000 Einwohner. Daher stellt die Forderung nach 2 internistischen Rheumatologen den Mindestbedarf dar. Inwieweit besonders geschultes Pflegepersonal oder auch moderne IT-Technik zur Bewältigung von höheren Patientenzahlen beitragen können, sollte modellhaft erprobt und evaluiert werden.

\section{Akutstationäre Versorgung}

Die Aufgaben der akutstationären Versorgung unterliegen kontinuierlichen Wandlungen, die sich u. a. durch den medizinischen Fortschritt, die gewünschte Verlagerung von Leistungen in den ambulanten Sektor und nicht zuletzt die demografische Situation ergeben. Letzteres bedingt einen eher zunehmenden Bedarf an akutstationärer Versorgung für ältere funktionseingeschränkte Patienten.

Eine Schätzung der benötigten akutstationären Kapazitäten ist nur mit Unschärfen möglich. Das 2008 veröffentlichte Memorandum hielt 48 Akutkrankenhausbetten für je 1 Mio. Ein- 
wohner für angemessen. Seither hat die Verweildauer in der internistischen akutstationären Rheumatologie weiter abgenommen, nicht zuletzt durch die Einführung der DRGs. Zudem achten die Kostenträger noch stärker auf die Indikationsqualität akutstationärer Aufnahmen. Andererseits besteht für spezialisierte rheumatologische Akutkrankenhausabteilungen die Möglichkeit der Durchführung der rheumatologischen Komplexbehandlung. Für Kliniken mit einem ambulanten Versorgungsangebot (\$ 116b SGB V-Ambulanz, Ermächtigungsambulanz, Hochschulambulanz, Institutsambulanz etc.) bestehen die Herausforderung und Chance, ambulant versorgte Rheumapatienten im Falle einer hier nicht mehr beherrschbaren Behandlungssituation direkt akutstationär weiter $\mathrm{zu}$ versorgen.

Aufgrund der jährlichen Diagnosestatistik des Bundesamtes für Statistik wurden für das Jahr 2013 im Auftrag des Verbands rheumatologischer Akutkliniken (VRA) die Zahlen stationär behandelter Patienten mit entzündlich rheumatischen Systemerkrankungen ermittelt [13]. Pro 100.000 Einwohner fanden 120 stationäre Behandlungen in diesem Indikationsgebiet statt. Bezieht man auch die Fälle ein, die fachfremd in anderen Abteilungen behandelt werden (s. unten), so kommt man auf eine Bedarfsberechnung ,akutstationäre Rheumatologie"von ca. 200 Patienten/100.000 Einwohner. Die durchschnittliche Verweildauer beträgt ca. 10 Tage und der Bettennutzungsgrad $85 \%$. Hieraus ergibt sich eine Bedarfszahl zwischen 39 Betten je 1 Mio. Einwohner (bei Zugrundelegung von 120 stationären Behandlungen) und 65 Betten je 1 Mio. (bei Zugrundelegung von 200 Behandlungen je 100.000 Einwohner). Der Mittelwert beider Zahlen ist 52. Wir korrigieren daher die 2008 ermittelte Bedarfszahl von 48 akutrheumatologischen Betten je 1 Mio. Einwohner nur minimal auf 50 Betten je 1 Mio. Einwohner (•Tab. 1).

Eine Erhebung des Verbands rheumatologischer Akutkliniken im Oktober 2016 ergab bei 41 antwortenden VRAMitgliedskliniken eine gesamte Bettenzahl von 1704, im Durchschnitt 42 Betten (Range 6-130). Die Gesamtzahl der dort tätigen internistischen Rheumatologen betrug 172, hinzu kamen 150 angehende Rheumatologen in Weiterbildung [24]. Die Versorgungsdichte betrug daher 1 Rheumatologe je 10 Betten oder 1 ärztlicher Mitarbeiter je 5,3 Betten. Von den 41 Kliniken verfügen 38 Einrichtungen über mindestens 2 hauptamtliche Rheumatologen, wie in der Strukturqualität 2010 für akutstationäre Rheumaeinrichtungen festgelegt [19]. Unterstellt man bei den 18 nicht antwortenden VRAKliniken und weiteren 20 rheumatologisch tätigen Krankenhausabteilungen in Deutschland, dass ihre Bettenzahl ebenfalls im Durchschnitt 42 beträgt, so kommt man auf weitere 1596 stationäre Betten, insgesamt also 3300 Betten. Diese Zahl ist, wie erwähnt, eine Hochrechnung. Die oben ermittelte Bedarfszahl von 50 Betten je 1 Mio. Einwohner ergibt 4000 Betten in ganz Deutschland. Dieser Mehrbedarf von ca. 700 Betten erklärt sich einerseits aus der Alterung unserer Gesellschaft und der steigenden Lebenserwartung von Patienten mit entzündlich rheumatischen Krankheiten, andererseits würde eine höhere Kapazität es auch ermöglichen, mehr Patienten mit entzündlich rheumatischen Erkrankungen fachspezifisch zu versorgen. In dem oben erwähnten Gutachten im Auftrag des VRA [13] wurde gezeigt, dass mindestens ein Drittel der Patienten mit dem Risiko eines schweren Krankheitsverlaufes verschiedener Rheumadiagnosen in internistischen Abteilungen ohne ausgewiesene Rheumafachabteilung akutstationär versorgt werden. Es ist davon auszugehen, dass viele dieser Patienten in einer Rheumaklinik angemessener versorgt wären, auch wenn bei einem Teil dieser Patienten die nichtfachspezifische Versorgung durch besondere Indikationen oder Komplikationen (z. B. Notwendigkeit intensivmedizinischer Versorgung) bedingt sein mag. Die Bedarfszahl von 4000 Betten für ganz Deutschland ist also unter der Prämisse des Abbaus nichtfachspezifischer stationärer Kapazitäten zu sehen.

\section{Medizinische Rehabilitation}

Die heute verfügbaren akutmedizinischen Therapiemöglichkeiten können auch zu einem veränderten Bedarf an Leistungen zur medizinischen Rehabilitation führen, dessen Schätzung ebenfalls nur angenähert möglich ist. Das DGRhMemorandum von 2008 ging am Beispiel der rheumatoiden Arthritis (RA) von 20.000 Leistungen pro Jahr im Zuge des Antragsverfahrens oder der Anschlussrehabilitation aus. Angesichts der zunehmend besseren funktionellen Kapazität der RA-Kranken [27] gehen wir von einem um $25 \%$ verringerten Bedarf, also 15.000 Leistungen pro Jahr, aus. Für die anderen entzündlich rheumatischen Erkrankungen muss ein ähnlicher Rehabilitationsbedarf wie für die RA unterstellt werden, sodass in der Summe bei 1,45 Mio. Betroffenen mit entzündlich rheumatischen Systemerkrankungen etwa die 2,6-fache Anzahl der für RA genannten Rehabilitationsleistungen indiziert ist, also ca. 40.000 p. a., wovon jeweils 20.000 Leistungen entsprechend dem gesetzlichen Auftrag in Trägerschaft der GRV bzw. der GKV durchzuführen sind.

Sofern die gesetzliche Rentenversicherung Träger der Maßnahme ist, sollte diese Versorgung in möglichst großen Kompetenzzentren für rheumatologische Rehabilitation (ca. 100 Betten bzw. ambulante Behandlungsplätze) erfolgen, die den Strukturvorgaben und Qualitätskriterien von BAR und DRV entsprechen [4,6]. Sie implizieren, dass viele Aufgaben der Rehabilitation vom multiprofessionellen Rehabilitationsteam mit Expertise für entzündlich rheumatische Erkrankungen wahrgenommen werden. Auf Basis dieser Strukturvorgaben und nach Einschätzung der Kommission sind für 40 Betten bzw. ambulante Behandlungsplätze mindestens 1 Rheumatologe und 1 Arzt in Weiterbildung vorzusehen. Die Einhaltung der Strukturvorgaben wird von den Leistungsträgern regelmäßig überprüft. Größere Einheiten sind auch deshalb notwendig, weil spezifische medizinische und berufsbezogene Angebote sowie Patientenschulung mit einer ausreichenden Anzahl von Personen mit ähnlichen Problemlagen stattfin- 
Hier steht eine Anzeige.

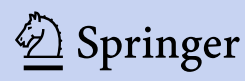


Tab. 1 Anhaltszahlen und Berechnungen

\section{Bedarfszahlen in der ambulanten Versorgung}

Prävalenz entzündlich rheumatische Krankheiten (2,1\%)

Inzidenz entzündlich rheumatische

Krankheiten (0,1\%)

Bedarf Rheumatologenkontakt präva- 4 x p. a. à 20 min

lente Fälle

Bedarf Rheumatologenkontakt inzidente Fälle

Mitbetreuung Arthrose, Osteoporose, Schmerzsyndrome (10\% von 18 Mio.)

Leistbar je Rheumatologe

Mindestbedarf

Ist-Zahl

Mehrbedarf in der ambulanten Versorgung

Bedarfszahlen in der stationären Versorgung

1. Akutstationäre Versorgung

Bedarf Bettenzahl ${ }^{a}$

Ist-Zahl (Hochrechnung)

Mehrbedarf akutstationäre

Rheumatologie ${ }^{a}$

Bedarf akutstationär tätige internistische Rheumatologen

Bedarf weitere ärztliche Mitarbeiter (Fachärzte oder Weiterbildungsassistenten)

Ärzte insgesamt (je nach Einrichtung, ohne Konsil, Lehre, Forschung)

Bedarf Rheumatologen bei 4000 Betten

2. Rehabilitative Versorgung

Bedarf GRV/GKV

Ist-Zahl GRV (Hochrechnung)

Ist-Zahl GKV (Hochrechnung)

Mehrbedarf stationäre und ganztags ambulante Rehabilitation GRV/GKV

Bedarf internistische Rheumatologen in der Rehabilitation

Bedarf weitere ärztliche Mitarbeiter

(Fachärzte oder Weiterbildungsassistenten)

Ärzte insgesamt

Mindestbedarf Rheumatologen bei 3200 Betten/Plätzen

Erw. erwachsene Einwohner, $E$ Einwohner, $h$ Stunden, GKV Gesetzliche Krankenversicherung, GRV Gesetzliche Rentenversicherung

aUnter der Prämisse des Abbaus von Fehlbelegungen in nicht rheumatologisch ausgewiesenen Einrichtungen
Häufigkeit und Bedarf

insgesamt in Deutsch-

land

1,45 Mio.

0,07 Mio.

$1,5 \times 40 \mathrm{~min}$

$2600 \times 15 \mathrm{~min}$

Max. 1760 h p.a.

1350 Rheumatologen

776 Rheumatologen

574 Rheumatologen

Pro 100.000 Einwohner

(E) bzw. Erwachsene

(Erw.)

2100/100.000 Erw. ${ }^{\text {a }}$

105/100.000 Erw.

2800 h/100.000 Erw. p. a.

$105 \mathrm{~h} / 100.000$ Erw.

$650 \mathrm{~h} / 100.000$ Erw.

5 Betten/100.000 E

3300 Betten

700 Betten

1 je $10-15$ Betten

1 je 10 Betten

1 je 5 bis 6 Betten

267 bis 400 Rheumatologen

$2 \times 20.000$ Leistungen p. a. $=3200$ Betten/Plätze

4 Betten/Plätze/100.000E

18.000 Leistungen p. $a .=$ 1440 Betten/Plätze

4000 Leistungen p. a. $=$ 320 Betten/Plätze

18.000 Leistungen p. a. = 1440 Betten/Plätze

1 je 40 Betten/Plätze

1 je 40 Betten/Plätze

1 je 20 Betten/Plätze

80 Rheumatologen den sollten. Im Bereich der gesetzlichen Krankenversicherung (v. a. Rentner) leisten wohnortnah an rheumatologische Akutkliniken angegliederte rehabilitative Einrichtungen einen nahtlosen Anschluss an die akutstationäre Rheumatologie. Dies können auch kleinere Einheiten sein. Entscheidend ist, dass die internistisch rheumatologische Sachkompetenz in der Einrichtung gesichert ist.

Nach der Statistik der Deutschen Rentenversicherung wurden im Jahr 2015 nach dem Antragsverfahren 7000 stationäre Rehabilitationsleistungen wegen rheumatoider Arthritis (RA), 2000 wegen anderer entzündlicher Polyarthritiden, 4100 wegen ankylosierender Spondylitis oder sonstiger entzündlicher Spondyloarthritiden und 2400 wegen SLE oder anderer Kollagenosen durchgeführt [6]. Anschlussrehabilitationen erfolgten deutlich seltener und wurden nur aggregiert angegeben. Angaben für ganztags ambulante Rehabilitationsleistungen wegen entzündlich rheumatischer Systemerkrankungen sind in der DRV-Statistik nicht ausgewiesen, nach anderen Datenerhebungen aber sehr viel seltener als stationäre Leistungen. Somit stehen dem dargestellten Bedarf von 20.000 Rehabilitationsleistungen der DRV wegen entzündlich rheumatischer Erkrankungen nach dem Antragsverfahren 15.500 stationäre sowie eine wahrscheinlich deutlich kleinere Zahl ganztags ambulanter Leistungen gegenüber, deren Anteil für diese Indikationsgruppe geringer geschätzt wird als für andere Krankheiten (14\% im Jahr 2015 [7], entsprechend 2500 Leistungen). Von den 20.000 erforderlichen Rehabilitationsleistungen der DRV werden 2000 nicht erbracht, für die bei Annahme einer durchschnittlichen Verweildauer von 23 Tagen in internistisch rheumatologischen Fachabteilungen der Rehabilitationseinrichtungen und einem Nutzungsgrad von 83 \% [29] im Jahr 2015 160 zusätzliche Betten bzw. ambulante Plätze benötigt werden. Die Kommission geht davon aus, dass sich die im Memorandum von 2008 beschriebenen deutlich größeren Defizite der rehabilitativen Versorgung im Zuständigkeitsbereich der gesetzlichen Krankenversicherung nicht 
verbessert haben. Bei einer mindestens um den Faktor 5 geringeren Erbringung von Rehabilitationsleistungen bei entzündlich rheumatischen Erkrankungen durch die Krankenkassen gegenüber der Rentenversicherung [25] werden mindestens 16.000 von 20.000 indizierten Maßnahmen durch die Krankenversicherung nicht erbracht. Die unter den oben genannten Prämissen der Verweildauer und des Nutzungsgrades fehlenden 1440 Betten bzw. ambulanten Plätze (1280 beim Kostenträger Krankenversicherung und 160 in Trägerschaft der Rentenversicherung) entsprechen bei einem Schlüssel von 1 Rheumatologen auf 40 Betten/Plätzen einem zusätzlichen Bedarf von 36 internistischen Rheumatologen.

\section{Differenz zwischen bedarfs- gerechter und tatsächlicher Versorgung}

\section{Ambulante Versorgung}

Am 31.12.2015 gab es in Deutschland in der ambulanten internistisch rheumatologischen Versorgung 665 Vertragsärzte oder angestellte Ärzte und 111 ermächtigte Rheumatologen [12]. Die Gesamtzahl von 776 Rheumatologen entspricht 1,1 Rheumatologen je 100.000 erwachsene Einwohner. Dabei gibt es erhebliche regionale Differenzen: So betrug die Zahl internistischer Rheumatologen in den Stadtstaaten Bremen, Berlin und Hamburg 1,6-2,1 je 100.000 Einwohner, während es in Rheinland-Pfalz und im Saarland 0,8 waren. Gegenüber der errechneten Bedarfszahl von mindestens 2 ambulant tätigen Rheumatologen je 100.000 Einwohner beträgt das Defizit also bundesweit 0,9 je 100.000 und regional unterschiedlich 0 bis 1,2. Die einzige Region, welche die Bedarfszahl erfüllt, ist Hamburg.

Wie im Abschn. „Ambulante Versorgung " bereits erwähnt, ist das tatsächliche Versorgungsdefizit als größer anzunehmen, weil wir bei der Berechnung der Bedarfszahl einerseits von sehr hohen Wochenarbeitszeiten, andererseits von in Vollzeit arbeitenden Rheumatologen ausgegangen sind. Wir haben die 111 ermächtigten, in Kliniken tätigen
Rheumatologen in vollem Umfang mitgerechnet. Hinzu kommt, dass 2015 von den 665 vertragsärztlich tätigen Rheumatologen 155 in der hausärztlichen Versorgung tätig waren [14], sodass nur 510 Vertragsärzte für die fachärztliche internistisch rheumatologische Versorgung zur Verfügung standen. Setzt man für die ermächtigten und die hausärztlich tätigen Rheumatologen $50 \%$ eines Vollzeitäquivalents an, so kommen wir auf 643 Vollzeitäquivalente internistisch rheumatologischer Versorgung oder 0,83 internistische Rheumatologen je 100.000 Einwohner.

\section{Akutstationäre und rehabilitative Versorgung}

Die Statistik des Bundesarztregisters weist 302 stationär tätige Internisten mit Schwerpunkt Rheumatologie und 139 Fachärzte für innere Medizin und Rheumatologie aus, insgesamt also 441 stationär tätige internistische Rheumatologen. Die 111 zur ambulanten Versorgung ermächtigten Rheumatologen sind hier mitgezählt. Ausschließlich stationär tätig, zum Teil auch in Rehabilitationseinrichtungen, sind daher 330 Rheumatologen.

Die obige Berechnung einer Mindestzahl von 1 internistischen Rheumatologen je 10 bis 15 rheumatologische Akutbetten (vgl. Abschn. „Akutstationäre Versorgung“) und einer Gesamtzahl von 4000 Betten kommt auf eine Bedarfszahl von 267 bis 400 in Vollzeit akutstationär tätigen internistischen Rheumatologen (ohne Weiterbildungsassistenten). Zusätzlich werden 80 in Vollzeit tätige internistische Rheumatologen in der stationären und ganztags ambulanten Rehabilitation in Leistungsträgerschaft der GRV oder GKV gebraucht (ohne Weiterbildungsassistenten) (vgl. Abschn. „Medizinische Rehabilitation“).

\section{Lösungsmöglichkeiten und Forderungskatalog}

Dieses Memorandum zeigt die Rahmenbedingungen für eine bedarfsgerechte rheumatologische Versorgung und legt Anhaltszahlen für die erforderliche Zahl in der Versorgung tätiger internistischer Rheumatologen vor.

In der ambulanten Versorgung benötigen wir 1350 internistische Rheumatologen, derzeit stehen 776 zur Verfügung. Es fehlt also fast die Hälfte der als Mindestzahl erforderlichen Ärzte. Dies hängt u. a. mit Beschränkungen der Niederlassung zusammen. Wir fordern daher eine eigene Bedarfsplanung für internistische Rheumatologen unabhängig von der Gesamtzahl der Internisten, da die Versorgungsleistung der internistischen Rheumatologen weder durch andere Fachinternisten noch durch Orthopäden mit oder ohne Zusatzbezeichnung Rheumatologie übernommen werden kann.

Ferner sind die Beschränkungen der Zahl abzurechnender Patienten und Leistungen für die Gruppe der internistischen Rheumatologen aufzuheben, da nur so kurzfristig das Versorgungsdefizit gemildert werden kann. Zur Umsetzung dieser bereits im Memorandum von 2008 erhobenen Forderung nach Aufhebung von Budget- und Leistungsgrenzen und der Schaffung einer sektorübergreifenden ambulanten Versorgung können nun bundesweit die ambulante spezialärztliche Versorgung und in einzelnen Bundesländern Selektivverträge beitragen.

Eine höhere Versorgungsleistung der vertragsärztlich tätigen internistischen Rheumatologen ist auch durch die abrechnungsfähige Delegation von Leistungen an medizinische Fachangestellte $\mathrm{zu}$ erreichen.

Ziel ist es, dass jeder Patient, bei dem der Primärarzt den begründeten Verdacht auf eine entzündlich rheumatische Erkrankung hat, innerhalb von 14 Tagen einen Termin bei einem internistischen Rheumatologen bekommt.

Um zu einer größeren Zahl internistischer Rheumatologen zu kommen, müssen auch die Weiterbildungskapazitäten ausgebaut werden. Die Zahl der derzeit weitergebildeten Ärzte reicht kaum aus, um die aus Altersgründen ausscheidenden Rheumatologen zu ersetzen. Eine Deckung des Bedarfs ist daher nur durch vermehrte Weiterbildung zu erreichen. Hier spielen die Akut- und Rehabilitationskliniken eine entscheidende Rolle. Darüber hinaus sollten Regelungen geschaffen und um- 
gesetzt werden, die den 155 in der hausarztinternistischen Versorgung tätigen Fachärzten für innere Medizin und Rheumatologie die Rückkehr in die spezialisierte fachärztlich rheumatologische Versorgung ermöglichen bzw. attraktiv machen.

Schließlich sind das Interesse der Studierenden an der Rheumatologie und die Kompetenz der jungen Ärzte auf diesem Gebiet zu steigern, um einerseits mehr Interessierte für eine internistisch rheumatologische Weiterbildung zu gewinnen, andererseits die Kompetenz der zukünftigen Hausärzte in der Früherkennung entzündlich rheumatischer Krankheiten und damit der sachgerechten Zuweisung zu erhöhen. Voraussetzung hierfür ist, dass es an jeder medizinischen Fakultät ein ausreichendes rheumatologisches Lehrangebot gibt, was bislang nicht der Fall ist [28]. Dies ist dauerhaft nur durch eigenständige Abteilungen bzw. Lehrstühle zu erreichen. Dieses Defizit in der universitären Primärausbildung trägt entscheidend zu den Defiziten auf allen anderen Ebenen bei.

Zusammengefasst ist daher Folgendes $\mathrm{zu}$ fordern und politisch umzusetzen:

1. Mindestens 1350 internistische Rheumatologen in der ambulanten Versorgung

Notwendige Maßnahmen:

- eigene Bedarfsplanung für die Zulassung von internistischen Rheumatologen zur vertragsärztlichen Versorgung, um die Verhältniszahl von 2 bis 3 Rheumatologen pro 100.000 erwachsene Einwohner, zu erreichen bei Aufhebung der Budget- und Leistungsgrenzen,

- bundesweit einheitliche abrechnungsfähige Delegation an geschulte medizinische Fachkräfte (wie in den Selektivverträgen mit einzelnen Krankenkassen derzeit exemplarisch umgesetzt),

- Rückgewinnung der derzeit hausärztlich tätigen internistischen Rheumatologen für die spezialisierte Versorgung (z. B. im Rahmen der ASV),

- flächendeckende Umsetzung einer sektorübergreifenden ambulanten rheumatologischen Versorgung auf
Grundlage des Basisbeschlusses des Gemeinsamen Bundesausschusses zur ambulanten spezialfachärztlichen Versorgung von rheumatischen Erkrankungen vom 15.12.2016 [10].

2. Sicherstellung des Nachwuchses an internistischen Rheumatologen Notwendige Maßnahmen:

- Schaffung von rheumatologischen Lehrstühlen oder selbstständigen Einheiten an allen medizinischen Fakultäten,

- Erhalt und Ausbau der Weiterbildungskapazitäten,

- spezifische Förderung der Weiterbildung im ambulanten und stationären Bereich analog der bestehenden bundesweiten Förderung der Weiterbildung zum Facharzt für Allgemeinmedizin.

3. Sicherstellung einer bedarfsgerechten akutstationären Versorgung

Notwendige Maßnahmen:

- Erhalt von rheumatologischen Akutkliniken mit nachgewiesener Struktur-, Prozess- und OutcomeQualität in einem Umfang von bis zu 4000 Betten, Abbau von Fehlbelegung.

4. Sicherstellung einer bedarfsgerechten rheumatologischen Rehabilitation

Notwendige Maßnahmen:

- Vorhaltung spezialisierter Einrichtungen mit einer Kapazität von 40.000 Maßnahmen p. a. und 3200 Betten bzw. ganztags ambulanten Plätzen.

5. Versorgung aller Patienten in Versorgungsnetzwerken

Notwendige Voraussetzungen:

- Federführung des internistischen Rheumatologen,

- bedarfsentsprechende Einbeziehung anderer Fachdisziplinen und nichtärztlicher Heilberufe,

- enge Kooperation mit Verbänden der Selbsthilfeorganisation Deutsche Rheuma-Liga bei Angeboten zur Stärkung von Autonomie und Partizipation der Betroffenen.

\section{Korrespondenzadresse}

\section{Prof. Dr. A. Zink}

Deutsches Rheuma-Forschungszentrum Berlin, Programmbereich Epidemiologie und Klinik mit Schwerpunkt Rheumatologie und Klinische Immunologie, Charité Universitätsmedizin Berlin

Charitéplatz 1, 10117 Berlin, Deutschland zink@drfz.de

Interessenkonflikt. A. Zink, J. Braun, E. GromnicaIhle, D. Krause, H.J. Lakomek, W. Mau, U. Müller-Ladner, J. Rautenstrauch, C. Specker, M. Schneider und die Kommission Versorgungsqualität der DGRh geben an, dass kein Interessenkonflikt besteht.

Dieser Beitrag beinhaltet keine von den Autoren durchgeführten Studien an Menschen oder Tieren.

\section{Literatur}

1. Berufsverband Deutscher Rheumatologen e.V. Versorgungslandschaft Rheuma (2016) Vertrag zur Versorgungslandschaft Rheuma nach $\S \S 140$ a ff. SGB V mit der Techniker Krankenkasse, der Barmer GEK und spectrumK. http://www.bdrh. de/kooperationsvertrag-vlr.html. Zugegriffen: 10 Febr. 2017

2. Braun J, Krause A, Aringer $M$ et al (2016) Europäische Versorgungsstandards für Menschen mit rheumatoider Arthritis. Übersetzung und Kommentierung der von der EULAR unterstützten Vorschläge des eumusc.net durch eine Task Force von DGRh und VRA mit Unterstützung der Deutschen Rheuma-Liga. Z Rheumatol 75:416-428

3. Braun J, Schneider M, LakomekHJ (2016) Eckpfeiler der Qualitätssicherung in der Medizin in Deutschland. Wichtige Impulse für die rheumatoide Arthritis.ZRheumatol 75:203-212

4. Bundesarbeitsgemeinschaft für Rehabilitation (BAR) (2016) Rahmenempfehlungen zur ambulanten Rehabilitation bei muskuloskeletalen Erkrankungen 2005. http:// www.bar-frankfurt.de/fileadmin/dateiliste/ publikationen/empfehlungen/downloads/ Rahmenempfehlung_muskuloskeletale Erkrankung.pdf.Zugegriffen: 10. Febr. 2017

5. Deutsche Gesellschaft für Rheumatologie KV (2008) Memorandum Rheumatologische Versorgung von akut und chronisch Rheumakranken. www.dgrh.de/dieDGRh/Archiv/ frühereVeröffentlichungen/Versorgung. Zugegriffen: 10. Febr. 2017

6. Deutsche Rentenversicherung (DRV) (2014) Strukturqualität von Reha-Einrichtungen - Anforderungen der Deutschen Rentenversicherung. http://www.deutscherentenversicherung.de/Allgemein/de/Inhalt/ 3_Infos_fuer_Experten/01_sozialmedizin forschung/downloads/quali_strukturqualitaet/ Broschuere_Strukturanforderungen.ZZugegriffen: 10. Febr. 2017

7. Deutsche Rentenversicherung Bund (2016) Rehabilitation 2015. Deutsche Rentenversicherung Bund, Würzburg

8. Fiori W, Franz D, Roeder N et al (2003) DRG-Evaluationsprojekt Rheumatologie. Abbildungsqualität und Anpassungsbedarf akutrheumatologischer 
Behandlungen im G-DRG-System. Schüling, Münster

9. Gemeinsamer Bundesausschuss (2016) Richtlinie des Gemeinsamen Bundesausschusses über die Bedarfsplanung sowie die Maßstäbe zur Feststellung von Überversorgung und Unterversorgung in der vertragsärztlichen Versorgung (Bedarfsplanungs-Richtlinie). Aktualisierte Version vom 15.10.2015, veröff.im Bundesanzeigervom 05.01.2016 B2, in Kraft getreten am 06.01.2016

10. Gemeinsamer Bundesausschuss (2016) Beschluss des Gemeinsamen Bundesausschusses über eine Änderung der Richtlinie ambulante spezialfachärztliche Versorgung $\S 116 \mathrm{~b}$ SGB V: Änderung der Anlage 1.1 - Ergänzung Buchstabe b (rheumatologische Erkrankungen). https://www.g-ba.de/ downloads/39-261-2826/2016-12-15_ASV-RL_ Ergaenzung-Rheuma.pdf.Zugegriffen: 10-2-2017

11. Gigerenzer G, Gray J (2013) Aufbruch in das Jahrhundert des Patienten. In: Gigerenzer G, Gray $\mathrm{J}$ (Hrsg) Bessere Ärzte, bessere Patienten, bessere Medizin, Bd. 2013. MWV, Berlin

12. Gesundheitsberichterstattung des Bundes (2016) Bundesarztregister. http://www.gbe-bund.de/ gbe10/trecherche.prc them rech?tk=14501\& tk2=15352\&p_uid=gast\&p_aid=94916203\&p_ sprache=D\&cnt_ut $=17 \& u t=15401$. Zugegriffen: 10. Febr. 2017

13. Jüngerkes $H$, Schlüter RG (2016) Analyse der akutstationären medizinischen Leistungsdaten rheumatologischer Krankheitsbilder. Krankenhausberatung, Düsseldorf

14. Kassenärztliche Bundesvereinigung (persönliche Mitteilung). Auswertung aus dem Bundesarztregister, Freigabedatum 15.10.2016.

15. Kassenärztliche Bundesvereinigung (persönliche Mitteilung). Auswertung aus dem Bundesarztregister, Freigabedatum 7.12.2016.

16. Kekow J (2014) Stationäre rheumatologische Versorgung in Deutschland. Z Rheumatol 73:135-138

17. Lakomek $H J$, Bungard $S$, Rudwaleit $M$ et al (2014) Das KOBRA-Qualitätsprojekt - Verleihung eines Qualitätssiegels an Einrichtungen der akutstationären Rheumatologie. Krankenhaus 10:914-923

18. Lakomek HJ (2006) Brauchen wir eine stationäre Rheumatologie? Dtsch Med Wochenschr 131:2292-2294

19. Lakomek HJ, Braun J, Gromnica-Ihle E et al (2011) Neufassung der Strukturqualität der akutstationären Rheumatologie - ein zukunftsweisendes Projekt. ZRheumatol 70:615-619

20. Lakomek HJ, Fiori W, Buscham K et al (2006) Zunehmend sachgerechte Abbildung der Rheumatologie im G-DRG-Fallpauschalensystem 2006. ZRheumatol 65:46-41

21. Lakomek HJ, Fiori W, Buscham K et al (2005) Die multimodale rheumatologische Komplexbehandlung (OPS-8-983) - Herausforderungen, Lösungen und Perspektiven. ZRheumatol 64:557-563

22. Lakomek HJ, Hülsemann J, Küttner $T$ et al (2009) Klinische Behandlungspfade in der akutstationären Rheumatologie - ein erfolgreicher Schritt zur Prozessökonomie. Arthritis Rheuma 29:343-352

23. Lakomek HJ, Neeck G, Lang B et al (2002) Strukturqualität akut-internistischer rheumatologischer Kliniken - Projektgruppenarbeit des VRA. ZRheumatol 61:405-414

24. Lakomek, J. (persönliche Mitteilung). Ergebnisse der Erhebung des Verbands rheumatologischer Akutkliniken im Oktober 2016 zur akutstationären rheumatologischen Versorgung.
25. Mau W, Beyer W, Ehlebracht-Konig I et al (2014) Leistungstrends der rehabilitativen rheumatologischen Versorgung in Deutschland. Z Rheumatol 73:139-148

26. Mau W, Beyer W, Ehlebracht-Konig I et al (2015) Treat to participation: DGRh-Positionspapier zur nachhaltigen Verbesserung des funktionalen Gesundheitszustandes von Rheumakranken. ZRheumatol 74:553-557

27. Mau W, Thiele K, Lamprecht J (2014) Trends der Erwerbstätigkeit von Rheumakranken. Ergebnisse aus Sozialversicherungsdaten und Kerndokumentation der Rheumazentren in Deutschland. ZRheumatol 73:11-19

28. Riemekasten G, Aringer M, Baerwald CGO et al (2016) Rheumatologie - Integration in die studentische Ausbildung (RISA). Zur aktuellen Struktur der internistischen Rheumatologie an deutschen Hochschulen (RISA III). Z Rheumatol 75:493-501

29. Statistisches Bundesamt (2016) Grunddaten der Vorsorge- oder Rehabilitationseinrichtungen 2015 - Fachserie 12 Reihe 6.1.2 2015. https://www.destatis.de/DE/Publikationen/ Thematisch/Gesundheit/VorsorgeRehabilitation/ GrunddatenVorsorgeReha.html. Zugegriffen: 10. Febr. 2017

30. Verein Zentrum Patientenschulung (2016) Patientenschulungsprogramme der DGRh. http://www. zentrum-patientenschulung.de. Zugegriffen: 10 . Febr. 2017

31. Zentralinstitut für die kassenärztliche Versorgung in Deutschland (2016) Zl-Praxis-Panel. Jahresbericht 2014. www.zi-pp.de/pdf/Jahresbericht_ 2014.pdf.Zugegriffen: 10. Febr. 2017

32. Zink A, Albrecht K (2016) Wie häufig sind muskuloskeletale Erkrankungen in Deutschland? ZRheumatol 75:346-353
Neue Therapie-Option

für schwer behandelbare RA-Patienten

Heutzutage kommt es bei rheumatoider Arthritis immer schneller zu einem Therapiewechsel, sollte keine deutliche Besserung erzielt werden. Dadurch kann vielen Patienten rasch geholfen werden. Andererseits gibt es Patienten, bei denen sich auch nach der zweiten oder dritten Biologika-Therapie - typischerweise mit TNF-Inhibitoren - keine wesentliche Besserung einstellt. Den Betroffenen erschließt sich mit dem neuen Wirkstoff Sirukumab nun eine neue Option.

Das ist das Ergebnis einer der bisher größten multizentrischen, weltweiten Studien zu schwer behandelbarer rheumatoider Arthritis mit dem neuen Wirkmechanismus, einer Interleukin-6-Zytokin-Blockade. „Das konnten wir in einer der bisher größten Studien-Populationen mit rund 900 Betroffenen in 35 Ländern zeigen. Diese Patienten zeigten trotz vorangegangener Biologika-Vortherapien eine anhaltend aktive Erkrankung. Für viele dieser Patienten waren die Therapiemöglichkeiten praktisch erschöpft. Mit Sirukumab konnte auch in dieser Patientengruppe die entzündliche Aktivität der Erkrankung deutlich gesenkt werden“, erklärt Daniel Aletaha von der Universitätsklinik für Innere Medizin III der MedUni Wien.

Die Wirksamkeit und Sicherheit von Sirukumab wurde in zwei unterschiedlichen Dosen (Injektionen zu je $50 \mathrm{mg}$ alle 4 Wochen bzw. $100 \mathrm{mg}$ alle zwei Wochen) untersucht. Dabei erzielte die 100 Milligramm-Dosierung eine etwas bessere Wirksamkeit. Die Zulassung des Medikaments könnte bereits in Kürze erfolgen. Gleichzeitig könnten die neuen Erkenntnisse zu Sirukumab dazu führen, dass auch Wirksamkeitsstudien bei anderen Entzündungserkrankungen gestartet werden, etwa bei anderen Arthritisformen oder anderen entzündlichen Erkrankungen, wie z.B. bei Vaskulitiden. Auch Indikationen außerhalb der Rheumatologie sind denkbar.

Medizinische Universität Wien (http://www.meduniwien.ac.at) 